\title{
BMJ Open Organisational perspectives on addressing differential attainment in postgraduate medical education: a qualitative study in the UK
}

\author{
Katherine Woolf, ${ }^{1}$ Rowena Viney, ${ }^{1}$ Antonia Rich, ${ }^{1}$ Hirosha Jayaweera, ${ }^{1,2}$ \\ Ann Griffin ${ }^{1,3}$
}

To cite: Woolf K, Viney R, Rich A, et al. Organisational perspectives on addressing differential attainment in postgraduate medical education: a qualitative study in the UK. BMJ Open 2018;8:e021314. doi:10.1136/ bmjopen-2017-021314

- Prepublication history for this paper is available online. To view these files, please visit the journal online (http://dx.doi. org/10.1136/bmjopen-2017021314).

Received 21 December 2017 Revised 8 February 2018 Accepted 13 February 2018

Check for updates

${ }^{1}$ Research Department of Medical Education, UCL Medical School, Royal Free Hospital,

London, UK

${ }^{2}$ Centre for Clinical Research in Neuropsychiatry, University of Western Australia, Crawley, Western Australia, Australia

${ }^{3}$ Research Department of Medical Education, UCL Medical School, London, UK

Correspondence to Dr Katherine Woolf; k.woolf@ucl.ac.uk

\section{ABSTRACT}

Objectives To explore how representatives from organisations with responsibility for doctors in training perceive risks to the educational progression of UK medical graduates from black and minority ethnic groups (BME UKGs), and graduates of non-UK medical schools (international medical graduates (IMGs)). To identify the barriers to and facilitators of change.

Design Qualitative semistructured individual and group interview study.

Setting Postgraduate medical education in the UK. Participants Individuals with roles in examinations and/ or curriculum design from UK medical Royal Colleges. Employees of NHS Employers.

Results Representatives from 11 medical Royal Colleges $(\mathrm{n}=29)$ and NHS Employers $(\mathrm{n}=2)$ took part $(55 \%$ medically qualified, $61 \%$ male, $71 \%$ white British/lrish, $23 \%$ Asian/ Asian British, 6\% missing ethnicity). Risks were perceived as significant, although more so for IMGs than for BME UKGs. Participants based significance ratings on evidence obtained largely through personal experience. A lack of evidence led to downgrading of significance. Participants were pessimistic about effecting change, two main barriers being sensitivities around race and the isolation of interventions. Participants felt that organisations should acknowledge problems, but felt concerned about being transparent without a solution; and talking about race with trainees was felt to be difficult. Participants mentioned 63 schemes aiming to address differential attainment, but these were typically local or specialty-specific, were not aimed at BME UKGs and were largely unevaluated. Participants felt that national change was needed, but only felt empowered to effect change locally or within their specialty.

Conclusions Representatives from organisations responsible for training doctors perceived the risks faced by BME UKGs and IMGs as significant but difficult to change. Strategies to help organisations address these risks include: increased openness to discussing race (including ethnic differences in attainment among UKGs); better sharing of information and resources nationally to empower organisations to effect change locally and within specialties; and evaluation of evidence-based interventions.
Strengths and limitations of this study

- Qualitative methods afforded in-depth understanding of the problem and how it is operationalised from the perspective of key stakeholders.

- Some participants had several roles across organisations, meaning we were able to gain perspectives from representatives of other relevant organisations, such as Health Education England.

- Qualitative methods and lack of random sampling mean that results are not statistically generalisable and introduce the possibility of selection bias; however, representation from across 11 UK medical Royal Colleges/Faculties/Academy of Medical Royal Colleges provided a breadth of organisational perspectives, complementing previous research with trainees and trainers.

- Low participation from the organisation NHS Employers meant we are not able to ascertain any differences between organisations responsible for training junior doctors and organisations responsible for employing them.

\section{INTRODUCTION}

It is well established that international medical graduates (IMGs), on average, have poorer academic and career progression compared with UK medical school graduates (UKGs), and black and minority ethnic (BME) doctors also have poorer outcomes compared with white doctors ${ }^{12}$ (see also Woolf $e t a l^{3}$ for a review). This differential attainment came into the spotlight in 2014 when the British Association of Physicians of Indian Origin brought the Royal College of General Practitioners and the General Medical Council (GMC) to judicial review over the low pass rates of IMGs in the Membership of the Royal College of General Practitioners Examination (MRCGP) ${ }^{4}$ following a 2013 review of the MRCGP commissioned by the $\mathrm{GMC}^{5}$ and a subsequent article by the review authors in the British Medical Journal. ${ }^{6}$ 
The current project is part of the GMC programme to understand and reduce differential attainment in UK medicine (http://www.gmc-uk.org/education/27486. asp). A 2015 rapid review on the topic commissioned by the GMC found that most research on differential attainment consisted of quantitative studies about high-stakes examinations; that examinations per se were not generally unfair; and although research was moving towards understanding 'the educational and social factors contributing to performance' (p. 45), there were still too few studies to draw firm conclusions. Finally, very few evaluations of interventions to reduce differential attainment were found. ${ }^{7}$

Following this review, the GMC commissioned University College London's (UCL) Research Department of Medical Education to undertake a qualitative study of stakeholder perceptions of the fairness of postgraduate medical training. In part 1 , we interviewed trainees and trainers across England and Wales in six specialties and foundation training about their perceptions of the fairness of postgraduate medical training. That work identified six risks to the progression of BME UKG and IMG trainees, and an additional six risks that only affected IMG (see box 1). ${ }^{3}$ In part 2, the current study, we explore how these risks are perceived by representatives of stakeholder organisations with responsibility for training and assessing doctors undertaking specialist training (medical Royal Colleges), and for the human resources aspects of employing doctors in training in England (NHS Employers).

The aim was to gain insight into organisational factors that may act as barriers or facilitators to addressing differential attainment. This is important since organisational factors predict job performance and satisfaction in general, ${ }^{8}$ and because a supportive organisational culture has been found to be crucial in facilitating IMGs' transition into UK clinical practice. ${ }^{9}$

Research questions were:

1. What are stakeholders' views on the risks to BME UKG and IMG trainees' progression?

2. How significant and amenable to change did they perceive the risks to be?

We also sought to examine the facilitators and barriers to implementing change, and to identify examples of interventions or actions in place to address differential attainment.

\section{METHODS}

\section{Participant sampling frame and recruitment}

The sampling frame included individuals with roles in examinations and/or curriculum design from 10 of the largest Royal Colleges (Psychiatrists, Surgeons, Radiologists, General Practitioners, Obstetricians and Gynaecologists, Anaesthetists, Paediatrics and Child Health, Physicians, Emergency Medicine, Pathologists) and the Academy of Medical Royal Colleges (AoMRC). It also included employees of NHS Employers with a remit
Box 1 Risks to the progression of UK medical graduates from black and minority ethnic group (BME UKG) and international medical graduate (IMG) trainees identified by trainees and trainers in Fair Pathways part 1. Reproduced from Woolf $e t a{ }^{\beta}$

Risks to the progression of BME UKG and IMG trainees

1. Poorer relationships with seniors and problems fitting in at work, sometimes because of unconscious bias, that can lead to fewer learning opportunities, lower confidence and increased chance of mental health problems.

2. Bias in recruitment, Annual Review of Competence Progression (ARCP) and at work could result in poorer outcomes.

3. Anxiety about potential bias could result in poorer outcomes.

4. Less autonomy in job choice resulting from poorer performance in examinations and recruitment can mean increased likelihood of being separated from family and support networks, and increased chance of mental health problems.

5. Fear of being labelled as problematic can impede trainees reporting or getting help for problems, including perceived racism.

6. Potential for lack of recognition from trainers about environmental stressors, especially because within medicine, there is a belief that failure results from lack of motivation or ability.

\section{Risks to the progression of IMG trainees only}

7. Inexperience with UK assessments, recruitment, UK cultural norms including communication and National Health Service/work systems.

8. Cultural differences can impede relationships with colleagues and potentially patients because of unfamiliarity with UK cultural norms, a feeling of not being understood by UKGs and because trainers can lack confidence in IMGs' prior training.

9. Lengthy time to learn cultural norms.

10. Potential stigma of supplementary help.

11. Anxiety about increased probability of examination failure.

12. Visa difficulties and costs, and ineligibility for jobs can reduce training opportunities.

around equality and diversity, education and training and workforce management.

Participants were recruited from within this sampling frame by inviting attendees of relevant events (resulting in participants from the Royal College of Ophthalmologists and the Faculty of Intensive Care Medicine), through website searches and emails to organisations asking for relevant contact details or names, through personal contacts and through snowball sampling (potential participants nominated colleagues to attend in their absence).

\section{Data gathering}

Participants were given an information sheet about the study, and then asked to read the executive summary of part 1 of the research which included the 12 risks to trainee progression. ${ }^{3}$ They then completed an online questionnaire, rating each risk for significance and amenability to change on a five-point scale (from very significant/very difficult to change to very insignificant/ very easy to change). Ratings were used as prompts within interviews, with participants being asked to explain their ratings and change them if they wanted. Interviews used 
a semistructured schedule to ensure consistency while allowing the exploration of particular areas of interest or importance raised by participants. Interview schedules are published on the GMC website. ${ }^{10}$ We allowed up to 2 hours for focus groups and an hour for interviews. When time was limited, we prioritised discussing the first six risks since they related to BME UKGs and IMGs whereas the second six related only to IMGs. Only researchers and participants were present during interviews.

To facilitate participation, focus group attendance was offered in person or online using Blackboard Collaborate video conferencing software. Interviews were conducted face-to-face or by telephone. Data collection was carried out by $\mathrm{HJ}$ (an Asian Australian female neuroscientist and medical student), AR (a white British female psychologist), RV (a white British female linguist) and KW (a white British female psychologist and medical educationalist). All focus groups and interviews were audio-recorded and professionally transcribed. Researchers took field notes. Data were collected at the Royal College of Physicians of London, the GMC offices in London, online and over the telephone.

\section{Analysis}

Thematic analysis ${ }^{11}$ was conducted using NVivo V.11@. ${ }^{12}$ $\mathrm{HJ}, \mathrm{AR}, \mathrm{RV}$ and $\mathrm{KW}$ read all transcripts, making notes. They met to jointly produce a first draft of the coding framework, which also referred to Mountford-Zimdars et al's analysis of similar data from UK higher education $^{13}$ in that it categorised levels of change at the micro (individual), meso (local) and macro (national) levels. Using the draft framework, all four researchers coded one focus group transcript independently, and then met again to refine the framework. HJ, AR and RV each coded one interview transcript with the revised framework and met again; however, since there were no disagreements, the framework was finalised. The transcripts were divided equally between the same three researchers who coded them independently, meeting regularly to discuss any areas of uncertainty and to ensure consistency. KW wrote the first draft of the results using the coded data. All authors agreed the final version.

The questionnaire responses were designed to prompt discussion rather than to be a statistically reliable representation of participants' views, and therefore are not presented.

\section{Ethics}

Participants gave their consent on the questionnaire and verbally at the start of the interview or focus group. Participants received a certificate of participation and those attending focus groups had refreshments.

\section{RESULTS}

\section{Participants}

Sixty-eight representatives from Royal Colleges and 56 from NHS Employers were invited; 31 participated:
29 from 11 Royal Colleges/Faculties/AoMRC and two from NHS Employers. Seventeen (55\%) participants were medically qualified, $19(61 \%)$ were male, $22(71 \%)$ were white British or white Irish, seven (23\%) were Asian/British Asian and two (6\%) were missing ethnicity data. Two people declined because of conflicting commitments or lack of time. Others did not give a reason. One person who was invited agreed to take part but then later did not respond to emails arranging an interview.

Data were gathered between September and December 2016 in five focus groups (FG1 to FG5) and seven interviews (I1 to I7). FG1, FG3, FG4, FG5 and I4, I7 discussed the first six risks only. All participants rated 12 risks in the questionnaire. The mean length of the interviews was $51 \mathrm{~min}$ (range: 31 to $76 \mathrm{~min}$ ), and the mean length of the focus groups was 1 hour and $47 \mathrm{~min}$ (range: 97 to 133 min).

Quotes are allocated to participants by referring to their participant number (P1 to P29), their ethnic group, gender and whether a medical doctor or not. Data from representatives of NHS Employers are not included since the participation rate was so low.

\section{Significance of risks}

Participants rated all risks as significant, although risks arising from being 'different' to the majority were perceived as most significant and those relating to trainee anxiety or stigma were perceived as least significant.

[Risk 7: IMG inexperience with UK systems and cultural norms] is the most significant risk I think, for people coming from different cultures. [...] Medical knowledge the same but [UK assessments, recruitment, UK cultural norms including communication and NHS systems] are different. And people know English language but they don't the nuances of English language. [...] It's quite a steep hill for them to climb. (P12 Asian/British Asian male medical)

[Regarding Risk 10: Potential stigma of supplementary help], it's difficult to see where the stigma comes from. Because it is common sense that, if you are an IMG, you are going to need a bit more help to get into it. And that should be accepted by both IMGs and their UK colleagues. (P10 white male non-medical)

All risks were felt to be more significant for IMGs than for BME UKGs with a small minority of (white male) participants questioning whether BME UKGs faced these risks:

In my experience, from the trainees I work with, the BME trainees are very good. [...] I've not seen anything specific in our hospital or in our Deanery where UK BME graduates are managed any differently $[\ldots]$ I wouldn't say that it is different for a UK white graduate or a UK BME graduate in the (region) of Scotland. (P13 white male medical) 
The importance of personal experience to perceptions of risk significance

When rating a risk's significance, participants drew mostly on evidence obtained through personal experience of interacting with and observing trainees, through evidence they had access to via their role (eg, examination scores, examinations appeals, supporting trainees 'in difficulty' or advising doctors employed in Trusts) or from equalities training they had received. Some female and Asian participants also discussed their own experience of being a member of a minoritised group. Evidence tended to be specific to the individual's specialty or local area.

I was talking to a BME trainee yesterday and I said 'What's your thinking... Have you ever, at any stage, felt that you've been discriminated against?' And he said to me, 'When I first came over here [...] I said to somebody, several people (that I) wanted to take Cardiology, and they looked at me and said "you've got no chance". It was as blatant as that, and the perception was 'Well, if you are from Pakistan you have no chance, you won't go onto Cardiology training school' . [...] But what he said in the exam is, 'Do you know something, we sometimes walk in and if we see a non-white examiner we will sometimes worry that they're going to be stricter'. [...] Purely anecdotal evidence from him. (P25 white male medical)

I've had just so much experience with [Risk 3] with candidates, and of course I look back on my own training as a BME candidate in my time. (P23 Asian/ Asian British woman medical)

Some participants recognised that their personal experiences were not necessarily generalisable and that their personal involvement could affect their objectivity, but generally participants did not think critically about the representativeness or accuracy of such evidence. For example, one participant (P16 white male medical Royal College) took the fact that he heard complaints from trainees and none were about racism as evidence that trainees did not have a problem reporting racism if it occurred. Furthermore, in general, a lack of evidence tended to lead to downgrading of significance. This was recognised by a few participants who made attempts to gather evidence to make informed decisions and persuade others to act.

Participants also referred to published research in considering the significance of risks. Research was highly valued and rarely critiqued; however, much of it was about examinations rather than other aspects of teaching, learning or assessment, meaning it was not relevant to most of the risks. Participants from Psychiatry, Medicine and General Practice seemed to feel more under scrutiny and pressure to deal with differential attainment and were particularly likely to refer to published research about their specialty, and actions underway to address differential attainment, whereas other specialties were still collecting data.

\section{Amenability of risks to change}

Participants were generally pessimistic about the possibility of change, and this was largely because they felt that the most significant risks required change at a macro (national) level, whereas they only had power to effect change at a meso (local) or micro (individual) level, although two participants described how top-down support from national organisations such as the GMC was important but not sufficient for change.

[Regarding Risk 12: Visa difficulties and costs, and ineligibility for jobs can reduce training opportunities] Immigration is a hot potato at the moment, since Brexit, before that. And the Government is trying to shut all doors for these people and so it's extremely, extremely difficult. It's a political football, it's difficult to change. (P12 Asian/Asian British male medical)

One exception was Risk 7 (IMG inexperience with UK systems and cultural norms), which while being perceived as highly significant was also rated as relatively easy to change, probably because it is well recognised at a national level and has many interventions in place to address it. It may also be because addressing it requires increasing trainees' knowledge which was felt to be easier than changing culturally bound attitudes and behaviours, which related more to Risks 1 and 8. Sometimes participants were pessimistic because they felt disempowered to effect change where it was needed outside their own organisation, such as this Royal College participant who felt change needed to occur within hospital Trusts, which he had no influence over:

I've had probably about five (examples) this week and thousands in the last couple of years of trying to convince (a) Trust to do seemingly incredibly straightforward things and they refuse. [...] It's 'No, we will not, we have a financial bottom line we have to keep to.' (P21 white male non-medical)

By contrast, a Royal College participant who also had a senior role within his Health Education England Local Education and Training Board (LETB) felt more positive about the ability of his Royal College to address Risk 6 (Potential for lack of recognition from trainers about environmental stressors) since his LETB was taking steps to address it:

[Risk 6] is definitely (something that) as an organisation, (my Royal College) and the LETB, (LETB name) can very much change. And I think, I know in (my LETB) we're trying to make all the education supervisors think about the context, so it's not just about an individual in the workplace struggling with an exam or patient feedback or clinical knowledge, there's health capabilities, professionalism, the wider pressures on somebody, their cultural issues, linguistic issues, personality issues. (P1 white male medical)

Participantsfeeling theylacked knowledge or evidenceespecially about the relative underperformance of BME 
UKGs-also made them less confident about effecting change.

It's difficult, isn't it, when you don't have concrete evidence of what you need to change. To respond to that. (P24 white female medical)

\section{Barriers to change}

We identified two additional barriers to change: sensitivities around race and the isolation of good practice. Participants recognised that concerns about appearing discriminatory in micro-level interactions with trainees could prevent trainers from helping BME and IMG trainees develop and learn effectively. At a meso level, although most participants felt that being open about differential attainment was a positive step, there was trepidation about being transparent about race-related problems, especially if those problems were not public knowledge. For example, one participant felt uncomfortable talking within the focus group about bias that had been identified within recruitment in his specialty, even though the problem had been recognised and addressed. He referred to talking about the issue within the focus group as:

Washing our-not dirty linen, but the linen we realised wasn't quite as clean as it could have been. (P16 white male medical)

Another participant in the same focus group from a different specialty later explained that his College was not investigating a potential cause of bias within their clinical examination:

(The College didn't) know how we get around that (problem) without opening up a whole can of worms that we shouldn't be opening. (P15 white male medical)

There was concern among some participants about positive action to help IMGs or BME UKGs. For example, although many participants believed Risk 4 was significant, they felt giving targeted support to BME or IMG trainees was unfair or perceived as unfair to white doctors.

P7: We accept people setting up, you know, a woman consultants group. Or a black and ethnic minority consultants group $[\ldots]$ You know, all of that is perfectly okay. The idea that you set up a white consultants group, mind you, it might would certainly cause... [laughter] ...or a male consultants group.

P9: Or a male support group.

P6: Middle-aged white men support group... [laughs]

P7: that meets at your golf club... [laughs]

P9: We've got one of those [laughs].

P7 White male medical

P9 White female medical

P6 White male non-medical
Regarding the isolation of good practice, participants often lacked access to examples of good practice from outside their specialty, which contributed to their lack of knowledge and feelings of disempowerment to address differential attainment locally. Variability in systems by geographic region, even within the same specialty, might also be a barrier, as discussed by two participants from the same specialty:

P1: I have seen bias at recruitment, I think, where correctly, perhaps, people are putting photos up to say 'if you know this person you can't assess them', but then actually people are making her laugh because they can't pronounce the name or there's some sort of comment about hairstyle or something.

P4: We don't do names. We do numbers.

P1: Well, exactly, but this is moderation, therefore selection is different across the country, as you're saying with ARCPs.

P4:So you do names in [recruitment], do you?

P1:Unless it's changed this year.

P4: No, we've had it for years. We have pictures of candidate 104.

P1: But it's still a picture, so it's comments.

P4: Still a picture, to make sure we're talking about the right person.

P1: And therefore I think that's putting bias into the assessors by even a giggle about the surname. It's unconscious. It's not intentional, but it'll have an effect.

P1 White male medical

P4 White female medical

\section{Interventions or actions currently underway to address differential attainment}

We recorded 63 examples of interventions or actions to address risks to the progression of BME UKGs and IMGs. We categorised them into: (1) training for trainers and examiners, (2) training and support for trainees, (3) leadership (eg, having a senior member of a Royal College with a particular role supporting IMGs), (4) transparency around data and engagement with stakeholders and (5) designing recruitment and assessments to minimise bias or unintended negative consequences. See Woolf et al for examples. ${ }^{10}$ Very few participants said interventions had been evaluated and no interventions were targeted at BME UKGs.

\section{DISCUSSION}

\section{Summary of findings}

Representatives of medical Royal Colleges recognised that BME UKGs and IMGs face significant risks to their progression in UK postgraduate training. Participants tended to downgrade risks they had not personally observed, not always explicitly recognising that their own experiences were not necessarily generalisable and that personal involvement can affect objectivity. The most 
significant risks were felt to be difficult to change since they required action at a macro (national) level, and participants only felt empowered to effect micro-level (individual) or meso-level (local) change. Perceived lack of knowledge to guide change was also a barrier. One exception was Risk 7 [IMGs' inexperience with UK systems and cultural norms] which was perceived as highly significant but also relatively easy to change, perhaps because it is widely recognised as a problem at a macro level, it has a number of interventions or actions in place to address it, and because addressing it requires increasing trainees' knowledge which is believed to be easier than changing attitudes and behaviours.

Other main barriers to change were sensitivities about race and the isolation of good practice. Participants believed that talking about race could impede trainee-trainer relationships and organisations were often wary of being transparent about race-related problems. Some felt that positive action to address inequalities was unfair to highly performing white UK graduates. The fact that good practice, research and data tended to be isolated within specialties and/or regions meant that participants often based their ratings of a risk's significance on their own personal experience rather than on generalisable data. It also meant that they could feel they did not have the relevant knowledge or power to act locally to effect change.

\section{Strengths and weaknesses of the study}

This is the first study to explore differential attainment among UK medical graduates from the perspective of representatives of organisations rather than individual teachers/trainers or students/trainees. The qualitative methodology provided valuable insights into the reasons why it has proved difficult to effect positive change despite differential attainment being known about since 1995, ${ }^{14}$ and provides insights into how to progress. Its qualitative methodology does not however provide statistically generalisable results.

The study had good representation from 10 medical Royal Colleges/Faculties plus from the AoMRC, and had a mix of people with various roles in assessment, curriculum design and recruitment, from both clinical and non-clinical backgrounds. There was relatively good representation of Asian groups and women, although none from black or other minority ethnic groups. There was very limited participation from NHS Employers, making it difficult to identify from the data any issues relating to the employment of trainees as distinct from their training. We are not certain why we had low participation from NHS Employers, and this may be of interest to explore in future research.

\section{Relationship to previous findings}

The fact that race is a taboo is well known. White people generally fear that if they bring it up they might offend or be accused of racism, whereas BME people fear being marginalised, stigmatised or blamed. ${ }^{15} 16$ For example, a BME trainee in part 1 of the current project ${ }^{3}$ described fearing the ramifications of even thinking about being the victim of racism:

No-one likes the one who's going to kick up a fuss or start saying 'Oh it's because I'm an ethnic minority this, that, and the other'. No you start getting yourself into problems if you start thinking like that. (Female, Asian Other, UK Medical Graduate, Core Medical Trainee)

Sensitivities around race are a recognised barrier to addressing the differential attainment present across higher education, which is described as 'a sensitive and highly politicised issue' (p. 30). ${ }^{17}$ Barriers include fear of instigating a counterproductive blame culture (blaming staff for poor teaching practices and BME students for relatively poor performance), concerns about lowering academic standards and fear that admitting problems concerning race and racism can damage an institution's reputation. A lack of recognition of the extent and seriousness of the issues can also be a problem, for example, Tolia-Kelly has written recently about how black academics complaining about the negative impact of racism at work are often dismissed as oversensitive. ${ }^{18}$

The isolation of good practice in addressing differential attainment reflects the specialty silos found in clinical medicine. As Hanauer explains, such silos can hinder understanding and prevent solutions being found: 'our view from these silos of expertise often misses or even ignores clues that relate to the aetiology and pathogenesis of diverse disorders' (p. 1). ${ }^{19}$ The relevance to differential attainment among UKGs is clear: despite being observed across specialties and settings, it is still largely addressed within specialties.

The fact that interventions were not targeted at BME UKGs and evaluation was not mentioned reflects findings of a review of interventions to help IMGs adjust to UK clinical practice, which found that none were independently evaluated. ${ }^{9}$ Similarly, Mountford-Zimdars $e t a l$ s review of the causes of differential attainment in UK higher education found that interventions to improve the attainment of BME UK students were rarely evaluated robustly. ${ }^{13}$

\section{Implications of the findings}

The lack of interventions to address differential attainment in UKGs probably results partly from difficulties talking about race. Differential attainment in IMGs is typically explained using a deficit model (eg, lack of knowledge and culturally appropriate skills), whereas ethnic differences in attainment among UK graduates are more difficult to explain away without reference to racism or discrimination, since BME UKGs have presumably attained a similar level of knowledge and skills as white UKGs. Regardless of the reason for it, the lack of targeted interventions is problematic, since differential attainment in UKG has persisted over decades and is widespread. Future research and interventions should address the issues faced by UK graduates separately from the issues faced by IMGs to ensure the former receives 
sufficient attention. Publishing evidence from rigorous independent evaluations of interventions is also vital.

In our view, to overcome the isolation of good practice, we must take a macro-level view of differential attainment. This includes exploring differential attainment in recruitment and selection, and in workplace-based assessments and training environments, as well as in examinations; exploring differences and similarities between specialties and regions; and examining the continuum of medical training from application to independent practice, and from excellence awards to fitness to practice complaints. National organisations whose remit spans specialties, such as the GMC, Health Education England, the AoMRC and Medical Schools Council, can facilitate sharing of data, research and initiatives. The administrative data cohort study UK Medical Education Database (www.ukmed.ac.uk) is one example of where this is already working. In addition, medical educationalists can look to UK higher education, where the Equality Challenge Unit recently introduced the Race Equality Charter Mark for universities, and to the National Health Service, which has the Workforce Race Equality Standard. Organisations implementing change would do well to heed Sara Ahmed's finding that an organisation's need to be seen by others to be performing well in terms of diversity can be a perverse barrier to meaningful change. ${ }^{20}$

\section{CONCLUSIONS}

Representatives from a variety of Royal Colleges recognised that BME UKGs and IMGS can face significant risks to their progression in postgraduate medical training. To effect positive change, those responsible for medical education and training should develop and evaluate interventions to address ethnic differences in attainment among UK medical graduates; find effective ways to manage individual and organisational sensitivities around race; and coordinate and disseminate research and good practice across specialties as well as undergraduate medical education.

Acknowledgements Thank you to the participants for giving up their time; to Dr Catherine 0'Keefe and Ms Lynne Rustecki of Health Education England and Dr Krishna Kasaraneni for advising on the data collection and dissemination; and to Professor Andrew Elder and the administrative team at the Royal College of Physicians London for helping us recruit participants and for providing rooms for two focus groups.

Contributors KW and AG designed the study with input from the General Medical Council. RV, AR, KW and HJ carried out the field work. RV, AR, HJ and KW analysed and interpreted the data with input from AG. KW drafted the manuscript and is the guarantor. All authors revised it critically for important intellectual content and approved the final version for publication. All authors agree to be accountable for all aspects of the work.

Funding The research was funded by the General Medical Council who were involved in designing the study, were kept informed of progress with the collection, interpretation and analysis of the data and approved this report before submission with grant number of GMC229. The researchers remained independent from the funders. A version of the findings was published on the GMC website in 2016.

Competing interests HJ had financial support from the General Medical Council who commissioned this research; KW receives a fee as educational consultant to the Membership of the Royal College of Physicians (UK) Examination.
Patient consent Detail has been removed from this case description/these case descriptions to ensure anonymity. The editors and reviewers have seen the detailed information available and are satisfied that the information backs up the case the authors are making.

Ethics approval Ethical approval was granted by UCL Research Ethics Committee (Ref: 0511/012).

Provenance and peer review Not commissioned; externally peer reviewed. Data sharing statement № additional data available.

Open Access This is an Open Access article distributed in accordance with the Creative Commons Attribution Non Commercial (CC BY-NC 4.0) license, which permits others to distribute, remix, adapt, build upon this work non-commercially, and license their derivative works on different terms, provided the original work is properly cited and the use is non-commercial. See: http://creativecommons.org/ licenses/by-nc/4.0/

(c) Article author(s) (or their employer(s) unless otherwise stated in the text of the article) 2018. All rights reserved. No commercial use is permitted unless otherwise expressly granted.

\section{REFERENCES}

1. Woolf K, Potts HW, McManus IC. Ethnicity and academic performance in UK trained doctors and medical students: systematic review and meta-analysis. BMJ 2011;342:d901.

2. General Medical Council. The State of Medical education and Practice in the UK. London: General Medical Council, 2013.

3. Woolf K, Rich A, Viney R, et al. Fair Training Pathways for All: Understanding Experiences of Progression. London: UCL, Education RDfM, 2016.

4. Queen's Bench Division. The Queen on the Application of BAPIO Action Ltd vs Royal College of General Practitioners and The General Medical Council, 2014

5. Esmail A, Roberts C. Independent Review of the Membership of the Royal College of General Practitioners (MRCGP) examination Manchester: University of Manchester, 2013.

6. Esmail A, Roberts C. Academic performance of ethnic minority candidates and discrimination in the MRCGP examinations between 2010 and 2012: analysis of data. BMJ 2013;347:f5662.

7. Regan de Bere SN S, Nasser M. Understanding differential attainment across medical training pathways: A rapid review of the literature. Plymouth: Plymouth University, 2015.

8. Peter L, John C. The effect of organisational culture and leadership style on job satisfaction and organisational commitment: A cross-national comparison. Journal of Management Development 2004;23:321-38.

9. Kehoe A, McLachlan J, Metcalf J, et al. Supporting international medical graduates' transition to their host-country: realist synthesis. Med Educ 2016;50:1015-32.

10. Woolf K, Rich A, Viney R, et al. Fair Training Pathways for All: Understanding Experiences of Progression. Part 2. London: UCL Medical School, 2016.

11. Braun V, Clarke V. Using thematic analysis in psychology. Qual Res Psychol 2006;3:77-101.

12. QSR International Pty Ltd. NVivo Version 11: QSR International Pty Ltd, 2016.

13. Mountford-Zimdars A, Sabri D, Moore J, et al. Causes of Differences in Student Outcomes. Bristol: Higher Education Funding Council for England, 2015.

14. Dillner L. Manchester tackles failure rate of Asian students. BMJ 1995;310:209.

15. Cooke L, Halford S, Leonard P. Racism in the medical profession: the experience of UK graduates. London: British Medical Association, Health Policy and Economic Research Unit, 2003.

16. Roberts JH, Sanders T, Wass V. Students' perceptions of race, ethnicity and culture at two UK medical schools: a qualitative study. Med Educ 2008;42:45-52.

17. Berry J, Loke G. Improving the degree attainment of Black and minority ethnic students Higher Education Academy and Equality Challenge Unit. London: Equality Challenge Unit, 2011.

18. Tolia-Kelly DP. A day in the life of a Geographer: 'lone', black, female. Area 2017;49:324-8.

19. Hanauer SB. A poor view from specialty silos. Nat Rev Gastroenterol Hepatol 2010;7:1-2.

20. Ahmed S. On being included: racism and diversity in institutional life. Durham, North Carolina: Duke University Press, 2012. 